\title{
inu \\ Optimization of Spot Efficiency of Double-Helix Point Spread Function and Its Application in Intracellular Imaging
}

\author{
Jun Yang ${ }^{1, *(\mathbb{D}, \text { Hanliang Du }}{ }^{1}$, Zhenhao Chai ${ }^{1}$, Lei Zhang ${ }^{1}$, Ben Q. Li ${ }^{2}$, Jianlei Cui ${ }^{1}\left(\mathbb{D}\right.$ and Xuesong Mei ${ }^{1}(\mathbb{D}$ \\ 1 State Key Laboratory for Manufacturing System Engineering, Xi'an Jiaotong University, Xi'an 710064, China; \\ dhl3606@stu.xjtu.edu.cn (H.D.); chaizh16@stu.xjtu.edu.cn (Z.C.); xjtuzlei@stu.xjtu.edu.cn (L.Z.); \\ cjlxjtu@mail.xjtu.edu.cn (J.C.); xsmei@xjtu.edu.cn (X.M.) \\ 2 Department of Mechanical Engineering, College of Engineering and Computer Science, \\ University of Michigan, Ann Arbor, MI 48128, USA; benqli@umich.edu \\ * Correspondence: softyj@xjtu.edu.cn
}

check for

updates

Citation: Yang, J.; Du, H.; Chai, Z.; Zhang, L.; Li, B.Q.; Cui, J.; Mei, X. Optimization of Spot Efficiency of Double-Helix Point Spread Function and Its Application in Intracellular Imaging. Appl. Sci. 2022, 12, 1778. https://doi.org/10.3390/ app12041778

Academic Editor:

Herbert Schneckenburger

Received: 3 December 2021

Accepted: 2 February 2022

Published: 9 February 2022

Publisher's Note: MDPI stays neutral with regard to jurisdictional claims in published maps and institutional affiliations.

Copyright: (C) 2022 by the authors. Licensee MDPI, Basel, Switzerland. This article is an open access article distributed under the terms and conditions of the Creative Commons Attribution (CC BY) license (https:/ / creativecommons.org/licenses/by/ $4.0 /)$.

\begin{abstract}
The nano-scale spatial positioning of nanoparticles in tumor cells can be achieved through the double-helix point spread functions (DH-PSF). Nevertheless, certain issues such as low light intensity concentration of the main lobes, the influence of the side lobes, and the aberrations of the imaging system result in poor image quality and reduce the positioning accuracy of the fluorescent nanoparticles. In this paper, an iterative optimization algorithm that combines Laguerre-Gaussian modes and Zernike polynomials is proposed. The double-helix point spread function, constructed by the linear superposition of the Laguerre-Gaussian mode and Zernike polynomials, is used to express aberrations in the imaging system. The simulation results indicated that the light intensity concentration of the main lobes is increased by $45.51 \%$ upon the use of the optimization process. Based on the simulation results, the phase modulation plate was designed and processed while a 4f positioning imaging system was built. Human osteosarcoma cells, labeled by CdTe/CdS/ZnS quantum dots, were used as samples, and the position imaging experiment was carried out. The image information entropy was used as the clarity evaluation index. The experimental results showed that the image information entropy of the DH-PSF position imaging was reduced from 4.22 before optimization to 2.65 after optimization, and the image clarity was significantly improved. This result verified the effectiveness of the optimization method that was proposed in this work.
\end{abstract}

Keywords: double-helix point spread function; iterative optimization; quantum dots labeling; cell optical imaging

\section{Introduction}

The continuous development of optical technologies has allowed for nano-scale measurements beyond the diffraction limitation [1]. This has led to nano-scale positioning measurements, giving rise to high-precision three-dimensional spatial positioning of fluorescent nanoparticles in cells. This technology has a major impact on the life sciences, drug design, and cancer treatment [2-5].

Through point spread function engineering, the spatial positioning of nanoparticles can be quickly and accurately realized [6]. The double-helix point spread function (DHPSF) is a special point spread function which is the superposition of a group of LaguerreGaussian modes [7]. By designing a special phase modulation plate, the double-helix spots image of nanopaticles can be obtained. There is a functional relationship between the rotation angle of the double-helix spots and the defocus distance of the nanopaticles. In this manner, the nano-scale spatial positioning of fluorescent nanoparticles can be realized [8]. DH-PSF composed of Laguerre-Gaussian mode superposition is widely used in spatial nanoparticle positioning $[1,9,10]$. However, a problem is that the light intensity of doublehelix spots is not fully concentrated in the main lobes but partially scattered to the side lobes, which results in poor imaging quality [11]. Moreover, during the imaging process, 
the optical system inevitably has aberrations that blur the double-helix spot image and affect the positioning accuracy [12].

To solve the problem of low light intensity concentration in the main lobes, Xiaoli Yin et al. added an axicon before the phase modulation plate to suppress the side lobes [13]. However, this method failed to solve the aberration problem of the imaging system. The best annular spiral phase plate was obtained by eliminating the side lobes of the vortex beam [14]. This was achieved by selecting an annular spiral phase plate with an appropriate width that used the different energy sources of the vortex beam, i.e., the main and side lobes $[15,16]$. However, because this method involves amplitude modulation, it can significantly reduce the light intensity utilization in the optical path and impose significant restrictions under the condition of weak light imaging. The adaptive optical aberration correction method [17] can effectively improve the aberration problems of the imaging system. However, it requires complex optical devices and algorithms to measure the wavefront, which induces a high cost and increases the complexity of the system, hindering broader use [18,19]. The aberration optimization algorithm based on deep learning [20] can solve this problem to a certain extent. However, training the neural network requires a large number of samples and data. For small-data research such as cell imaging, errors can be large. The uncertainty level can be increased, and the applicability is limited [21]. The aberration correction optimization algorithm, which is based on the particle swarm optimization algorithm, obtains the optimal solution by adjusting the weight of each item of Zernike polynomials and taking the image information entropy as the evaluation standard of image sharpness [22,23]. However, this algorithm cannot ensure the double-helix characteristics of the modulation function, which means the functional relationship between the defocus distance and the double-helix spots rotation angle in the optimization process.

Thus, adopting the Laguerre-Gaussian mode and Zernike polynomial as the core techniques, and based on an iterative algorithm, this paper proposes a DH-PSF optimization algorithm to obtain the optimized phase modulation plate. The imaging quality was compared before and after processing optimization by building a $4 \mathrm{f}$ positioning optical path system. Simulations and experimental results indicated that the optimization method proposed here could increase the main lobes' light intensity concentration by more than $45 \%$, and could significantly improve the imaging quality.

\section{Optimization of the Positioning Method of the Nano-Scale Double-Helix Point Spread Function}

\subsection{Optimization of Double Spot Main Lobes Efficiency}

As shown in Figure 1, the light intensity distribution of the double-helix spots consists of the main lobes and several side lobes. The main lobes refer to the two spots in the middle where the light intensity is at its maximum (as shown in Part a). The side lobes refer to the corrugated secondary light spots with lower intensity (as shown in Part b). The side lobes' intensity is gradually weakened outside of the main lobes, and the larger the topological charge of the Laguerre-Gaussian mode is, the larger the side lobe becomes [24-26].

Thus, based on the optical characteristics analysis of the image and the definition of the main lobe efficiency formula, this work proposes an iterative scheme to optimize the Laguerre-Gaussian mode. The core idea is to optimize the weight of each LaguerreGaussian mode through iteration. The purpose is to reduce the influence of the side lobes and the attendant aberrations on the energy concentration of the main lobe without affecting the characteristics of the defocus double helix.

Analyzing the spiral double-spot shows that the main lobe distribution can be approximately considered as a Gaussian spot formed by the point light source which passes through the imaging system. Therefore, two Gaussian spots centered on the central point of the main lobe are selected as the final optimization goal. 


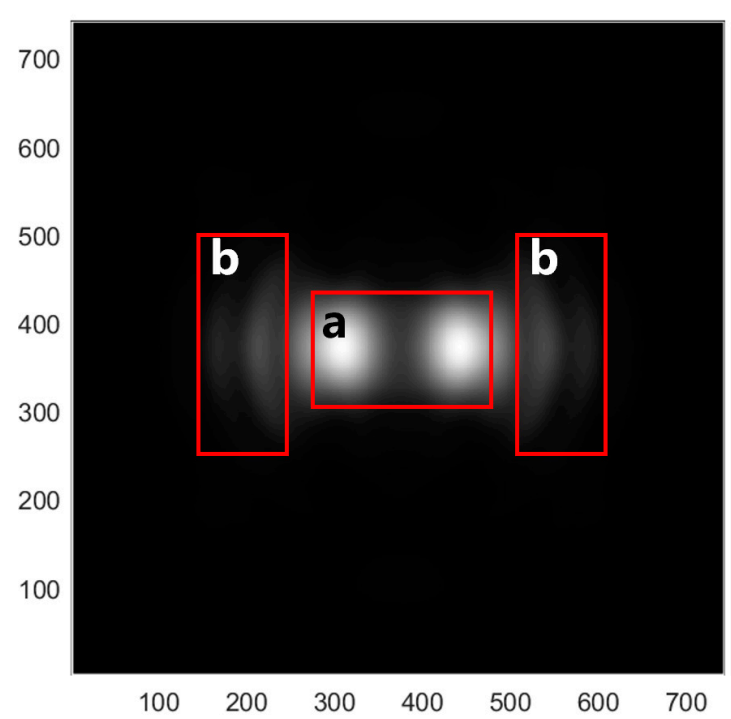

Figure 1. The simulation image of double-helix spots (a) the main lobes; (b) the side lobes.

The energy efficiency of the main lobes refers to the proportion of the light intensity of the main lobes to the overall light intensity.

Its calculation formula is

$$
\eta_{\text {main }}=\frac{I_{\text {main }}}{I_{\text {total }}}
$$

where $I_{\text {main }}$ represents the light intensity of the main lobes and $I_{\text {total }}$ represents the overall light intensity.

In the iterative algorithm, a group of defocus planes with a defocus distance of -2 to +2 $\mathrm{mm}$ were selected as optimization objects to ensure that the defocus rotation characteristics of the double spot did not change. The average main lobe efficiency refers to the average of the main lobe efficiency corresponding to these defocus distances.

Its calculation formula is

$$
\bar{\eta}=\frac{\sum_{j=1}^{K} I_{\text {mainj }}}{K}
$$

where $I_{\text {mainj }}$ represents the main lobe efficiency at the $j$ defocusing distance, and $\mathrm{K}$ represents the total $\mathrm{K}$ main lobe efficiency.

To facilitate calculation, the main lobes were regarded as the same point light source to form two Gaussian light spots with the maximum light intensity of the main lobes as the center. The rest were calculated as side lobe intensity.

The derivation process is as follows: according to [27], the expression of different Laguerre-Gaussian modes after linear superposition is

$$
\mathrm{S}_{k}(r)=\sum_{J=1}^{Q} A_{j_{k}} u_{m_{j} n_{j}}(r)
$$

where $k$ represents the $k^{\text {th }}$ iteration, $A$ represents the weight coefficient of the LaguerreGaussian mode, and $Q$ is the number of Laguerre-Gaussian modes. Then, the light field distribution of the point light source on the image plane can be expressed as

$$
U_{i}\left(x_{i}, y_{i}\right)=\frac{1}{i \lambda f} \iint U_{s p p}\left(x_{s p p}, y_{s p p}\right) S\left(x_{s p p}, y_{s p p}\right) \times \exp \left[-i \frac{2 \pi}{\lambda f}\left(x_{i} x_{s p p}+y_{i} y_{s p p}\right)\right] d x_{s p p} d y_{s p p}
$$

The respective strength distribution can be simplified, leading to

$$
I_{1 k}(x, y)=\left|U_{k}\right|^{2}
$$


The aberrations of the system are expressed as the sum of Zernike polynomials, and their expression is

$$
\phi(u, v)=\sum_{j=1}^{K} C_{j} Z_{j}(u, v)
$$

The final light intensity distribution at the image plane is

$$
I_{2 k}(x, y)=I_{1 k} \otimes \phi(u, v)
$$

The aforementioned analysis indicates that the ideal light intensity distribution is the Gaussian spot formed by the point light source that focuses on the focal point, and its size range is fixed. Therefore, if the ideal light intensity distribution is $C$, the deviation between the actual light intensity distribution and the ideal value is

$$
\Delta I_{k}=I_{2 k}-C
$$

Taking this deviation as an influencing factor, combined with the Laguerre-Gaussian modes under various parameters, the iterative formula which is obtained can be written as

$$
A_{j_{k+1}}=\sum\left(\Delta I_{k} \sum_{j=1}^{Q} u_{m_{j} n_{j}}(r)\right)
$$

Based on Equation (7), the iterative algorithm flow is shown in Figure 2. Since the microscope used in the actual measurement process magnifies the object by a factor of 100 times, the range of a single measurement is $\pm 2 \mu \mathrm{m}$. To keep the spiral characteristics of the modulation function unchanged, 10 defocus planes with equal intervals between -2 and $+2 \mathrm{~mm}$ are selected as the optimization objects.

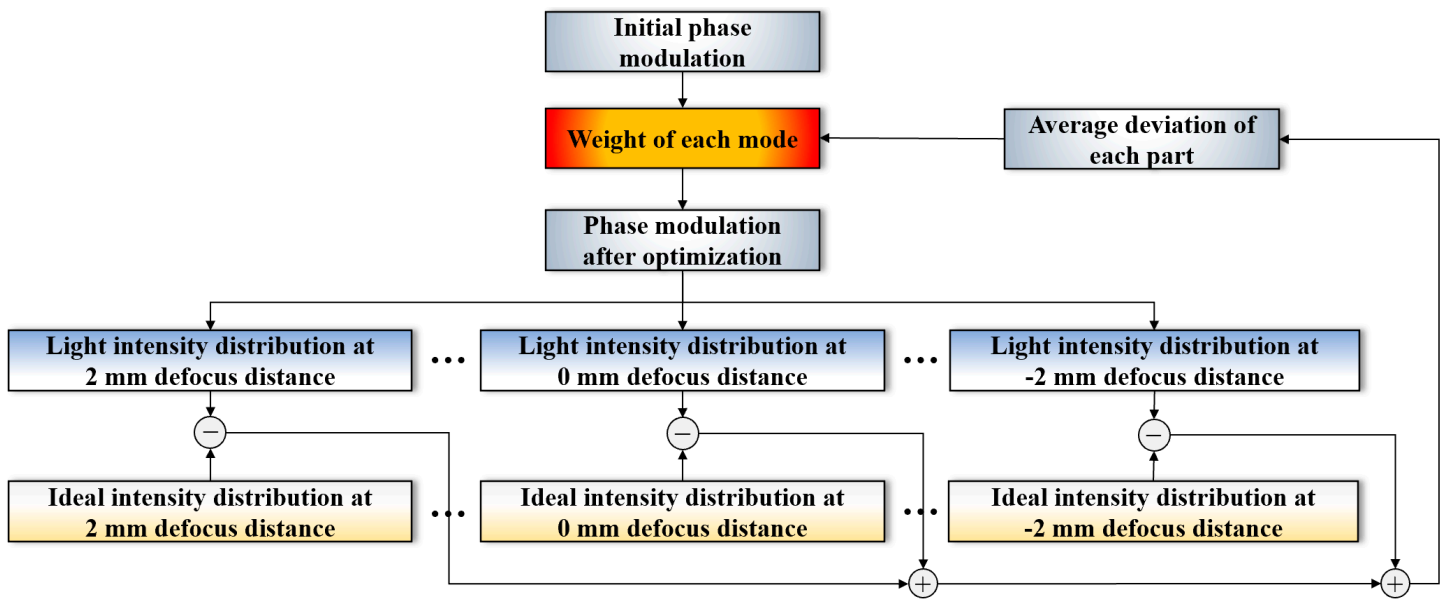

Figure 2. Iterative algorithm flow.

\subsection{Algorithm Simulation and Optimization Results}

At the end of each iteration, the algorithm computes the average efficiency of the main lobes, i.e., the ratio of the main lobe energy of the spot over the total energy of the light intensity distribution, and compares it with the previous iteration. If the efficiency converges, the iteration ends. As shown in Figure 3, before the iterative optimization, the weight of each Laguerre-Gaussian mode was 1 , and the average efficiency of the main lobe was $35.44 \%$. After the first iterative optimization, the average efficiency of the main lobes was increased to $38.77 \%$ and the variation range was obvious compared to the initial average efficiency. After the 19th iteration, the average efficiency saturates to a stable value ranging from 41.7 to $42.2 \%$. The maximum value is $42.02 \%$, which is $18.57 \%$ higher than the one prior to the optimization. After the algorithm implementation, the new weight value 
of each Laguerre-Gaussian mode is obtained. Due to the double-helix characteristic of the point spread function, which is determined by its superimposed Laguerre-Gaussian mode, the contribution of each part to the sidelobe energy is different [24]. Therefore, according to the order of magnitude of the new weight value, the initial weight is adjusted before the optimization, keeping its double-helix characteristics unchanged, and, subsequently, the iterative algorithm is executed again. The variation trend observed is similar. At the 19th iteration, the average efficiency of the main lobe converges, and the algorithm is terminated. By this time, the average efficiency of the main lobe of the spot remains stable between 51.3 and $51.8 \%$, with a maximum of $51.57 \%$. Compared to the average efficiency of the main lobes before the optimization process, the efficiency was improved by $45.51 \%$.

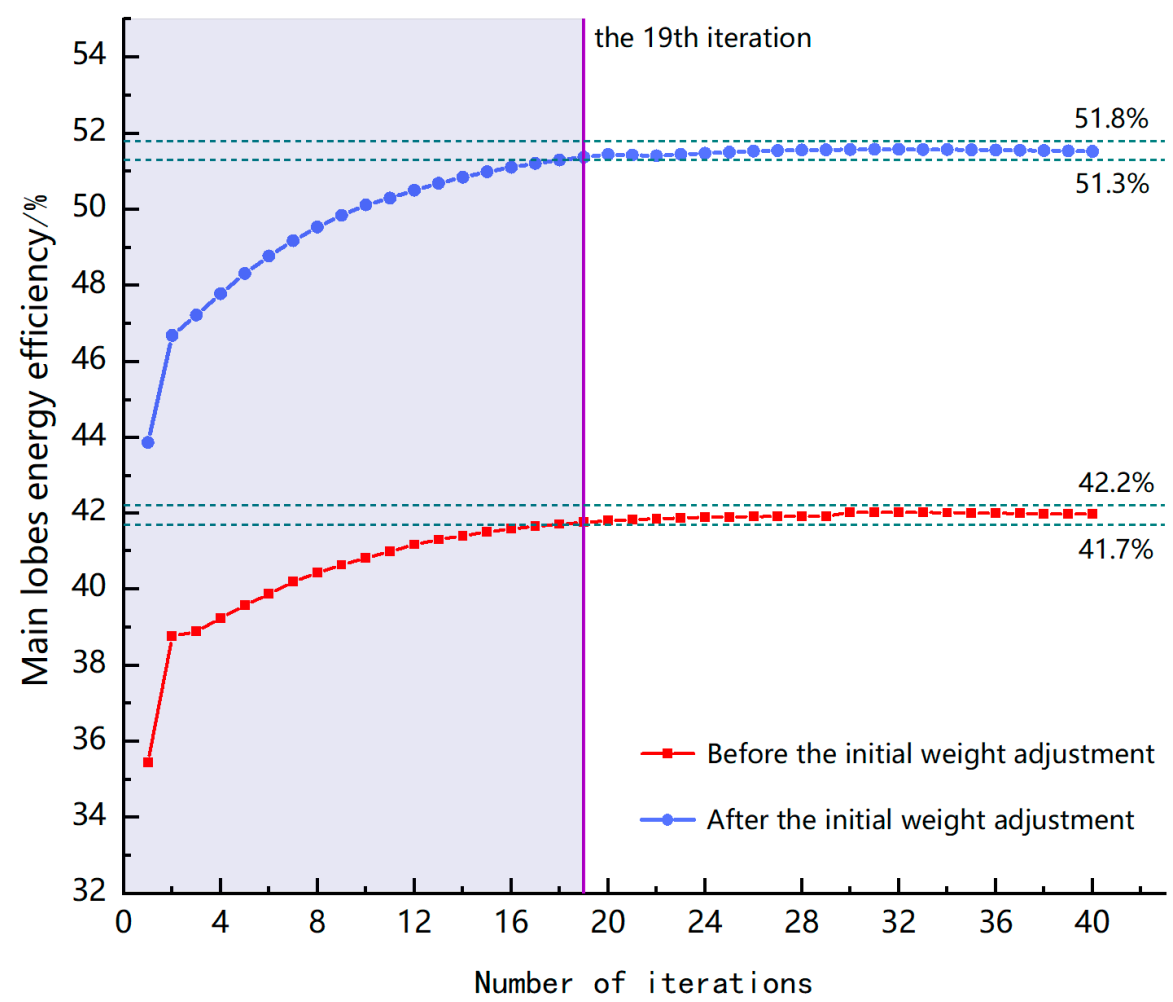

Figure 3. Iterative optimization results.

The CPU is an Intel Core i5-1135G7@2.4GHz (Intel, Santa Clara, CA, USA) and the memory is $16.0 \mathrm{~GB}$. Two rounds of iterative optimization are required according to the iterative optimization algorithm proposed in this paper. After the 19th iteration, the average efficiency of the main lobes converged, and the algorithm ended with a time of $1355.072190 \mathrm{~s}$. The two rounds took a total of $2710.14438 \mathrm{~s}$.

After the iterative optimization algorithm, the phase modulation function is shown in Figure 4. Figure 4a depicts the phase modulation function before the optimization, and Figure $4 \mathrm{~b}$ shows the phase modulation function after the optimization. The overall structure of the optimized phase modulation function is the same as the one before the optimization, indicating that the double-helix property of the modulation function does not change significantly. Note that the ripple pattern in the center is significantly reduced and the phase variation of each part is gentler. 

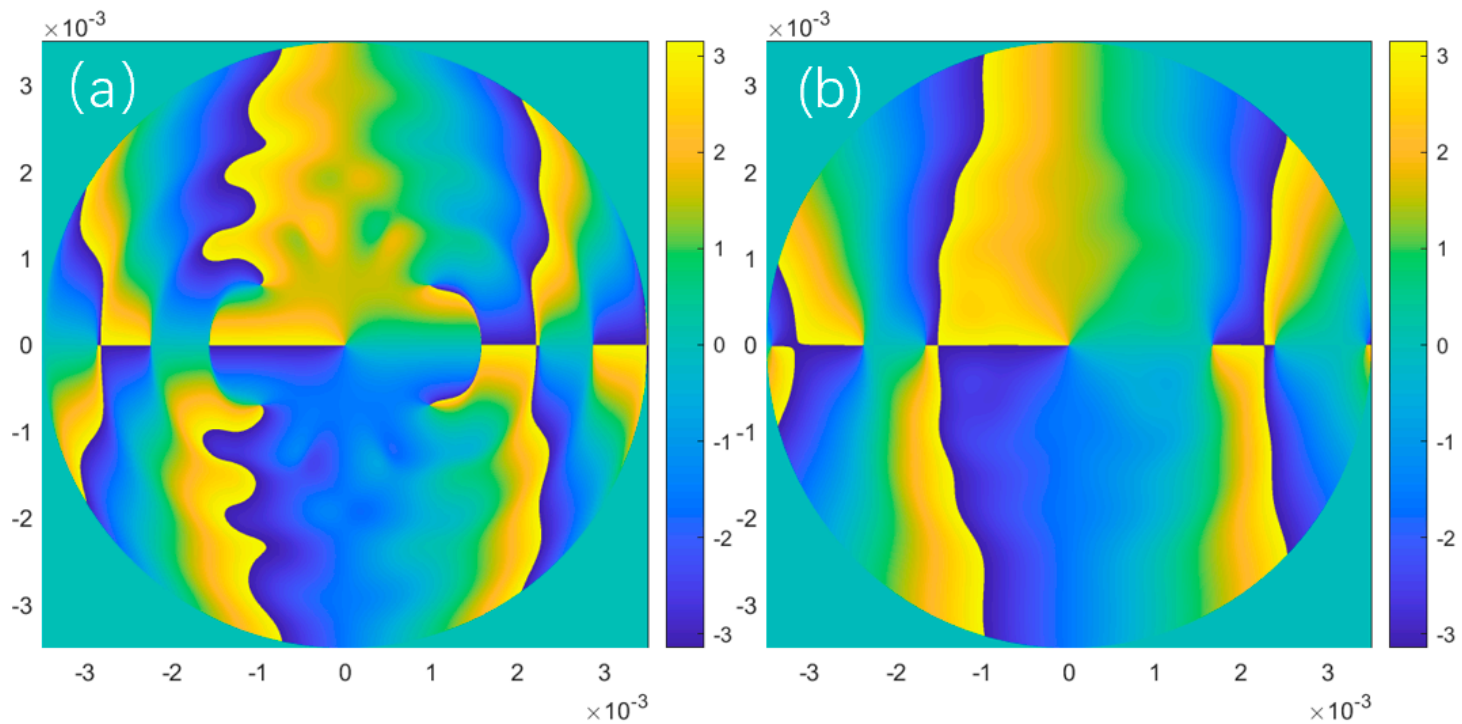

Figure 4. (a) Phase modulation function before optimization; (b) phase modulation function after optimization.

In the iterative process, the double-helix characteristics of the modulation function remain unchanged. Figure $5 \mathrm{a}-\mathrm{c}$ illustrate the double spot patterns for defocus distances of $-2,0$ and $2 \mathrm{~mm}$ before optimization. Similarly, Figure 5d-f illustrate the double spot patterns at the same defocus distances after optimization. Figure 5 also shows that the double spot rotation angle at the corresponding defocus distance does not change before and after the optimization, indicating that the iterative optimization process has no impact on the double-helix characteristics of DH-PSF.
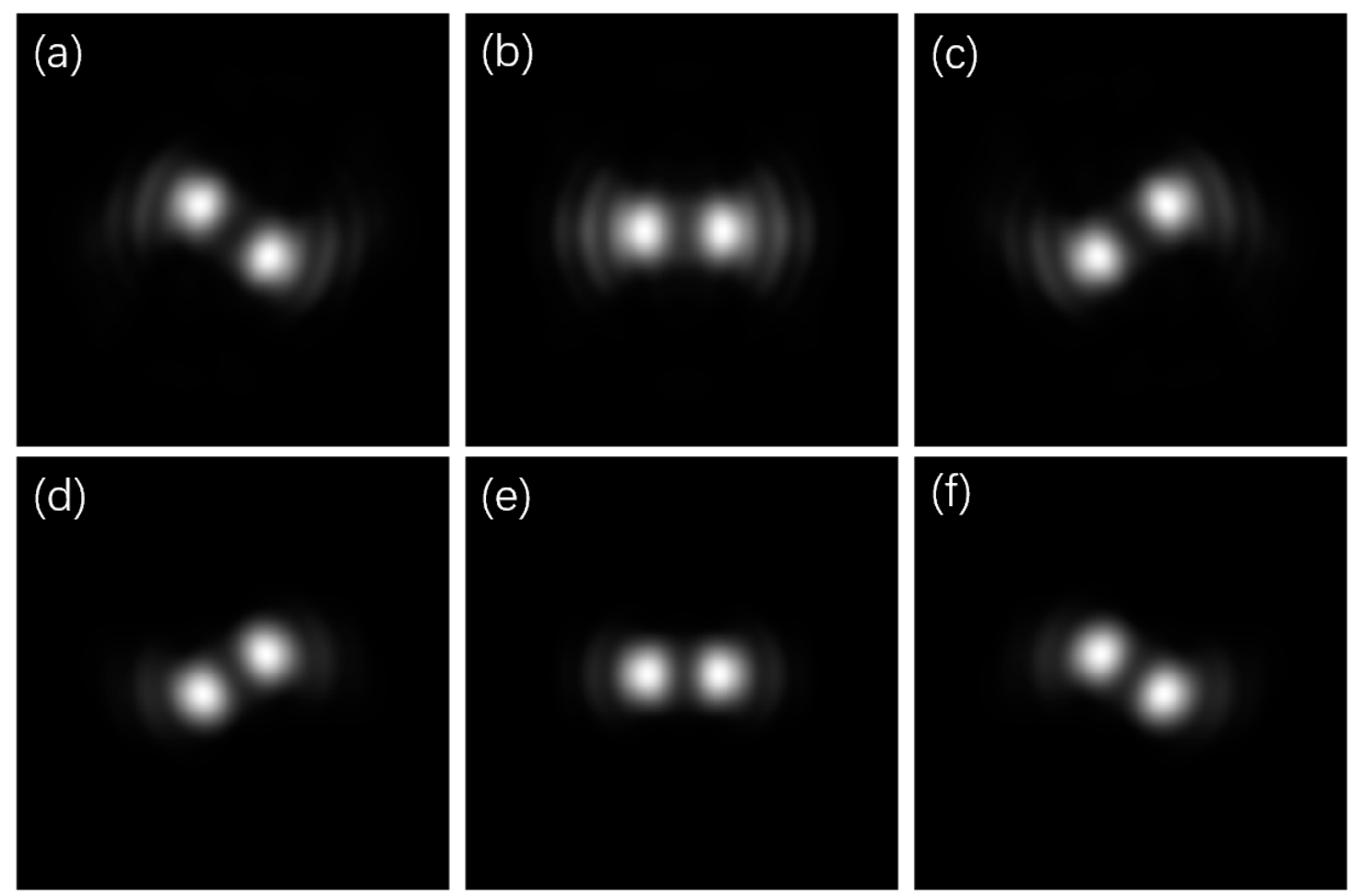

Figure 5. Optimize the double spot pattern at the defocus distance of $-2,0$, and $+2 \mathrm{~mm}$ before and after: $(\mathbf{a}-\mathbf{c})$ are double spot patterns before optimization; $(\mathbf{d}-\mathbf{f})$ are double spot patterns after optimization. 
Figure 6 shows the double spot pattern and the corresponding light intensity distribution curve at a defocus distance of $0 \mathrm{~mm}$. Through the iterative optimization process, the main lobes of the double spot become more rounded, the connection between the spots becomes weaker, the stray light range of the side lobes decreases, and the energy intensity is lowered. The efficiency of the main lobe of the spot increased from 35.07 to $53.26 \%$. The energy dispersion induced by the side lobe is significantly reduced. After the optimization process, the main lobes become clearer.
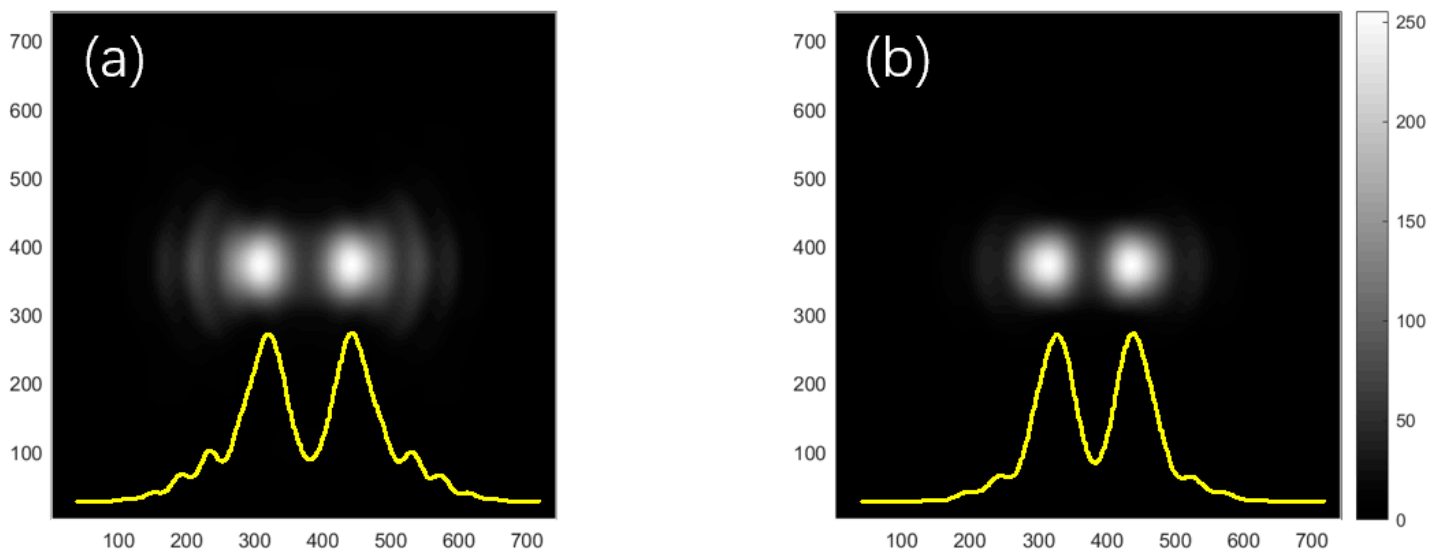

Figure 6. Before and after optimization, the double-helix spot simulation pattern at the focal plane: (a) The simulated double spot pattern and the corresponding intensity distribution curve before iterative optimization; (b) the simulated double spot pattern and the corresponding intensity distribution curve after iterative optimization.

\section{Experiment and Analysis}

The three-dimensional spatial localization of quantum dots in tumor cells is realized by using CdTe/CdS/ZnS as a fluorescent probe to label human osteosarcoma cells and a positioning light path with DH-PSF. The schematic diagram of the DH-PSF $4 \mathrm{f}$ positioning optical path system is shown in Figure 7. The laser transmitter generates a beam which triggers the quantum dots inside the cell to produce fluorescence. Then the fluorescence is amplified and captured by the microscope system. Subsequently, the imaging beam enters the $4 \mathrm{f}$ positioning light-path system and is modulated by a double-helix point spread function. A double-helix spot image is generated and received by the camera. Finally, it is transmitted to the computer system for further analysis.

In the first place, tumor cells are labeled by CdTe/CdS/ZnS quantum dots, which were transfected by lipo2000 liposomes. The tumor cells used in this experiment were human osteosarcoma cells (HOS). The complete medium used for culturing cells was made from Dulbecco's modified eagle medium (DMEM) basic medium, 10\% fetal bovine serum, and $1 \%$ double antibody solution. The tumor cell samples, labeled with CdTe/CdS/ZnS quantum dot probes, were captured and measured on the imaging system platform. The tumor cell samples which were labeled by the quantum dot probes were placed on the objective table of microscope and the excitation of the laser light source was irradiated to the sample through the lower objective lens. The corresponding image was captured by a fluorescence microscope (Olympus IX53, Tokyo, Japan).

Finally, by adjusting the imaging field, both the bright field and the fluorescence images of a single tumor cell were obtained, as depicted in Figure 8. 


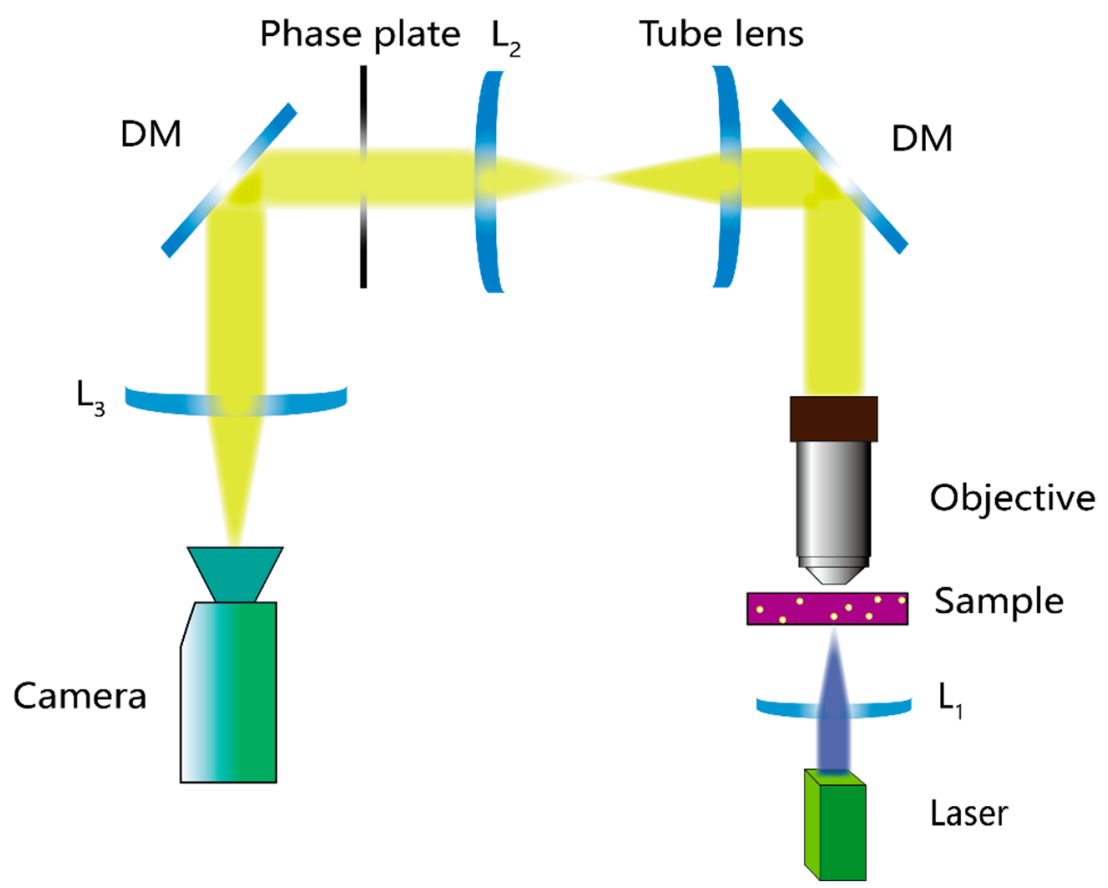

Figure 7. Schematic diagram of DH-PSF $4 \mathrm{f}$ positioning optical path system.

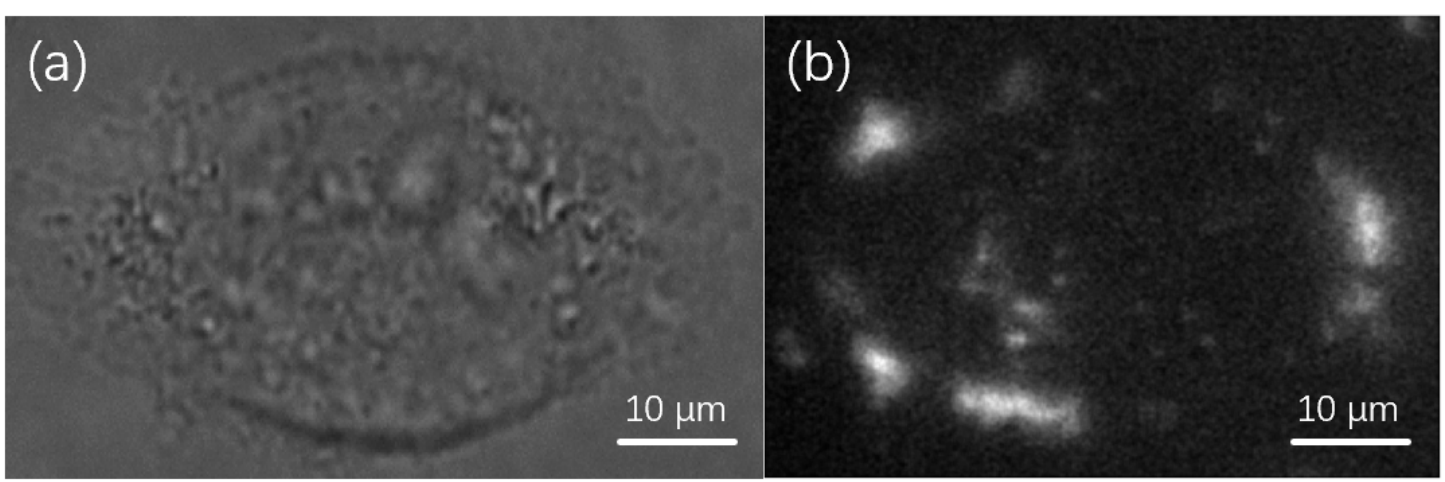

Figure 8. The experiment of cell labeling: (a) the bright field image; (b) the fluorescence image.

Two phase modulators were installed in the DH-PSF positioning light-path system. The first was made by using the optimized algorithm proposed in this paper, and the other was made by using the non-optimization algorithm. The actual images of quantum dots in tumor cells were obtained by modulating the fluorescence imaging of the cells, respectively.

The image clarity index of the final imaging results was evaluated by the image information entropy [23], which is

$$
E(x, y)=-\sum_{x, y} h(x, y) \log [h(x, y)]
$$

With $h(x, y)$ representing the acquired image matrix.

According to Bi et al. [23], the smaller the image information entropy is, the less information is required for image restoration, which means the image is clearer.

Figure 9 shows the localization images of the quantum dots in the tumor cells with and without optimization, respectively. The calculation shows that the image information entropy with and without optimization was 2.65 and 4.22, respectively. It becomes apparent that the image definition is significantly improved by the optimization process, as the image information entropy is reduced by $37.20 \%$. As a result, the findings of this study strongly 
suggest that the proposed iterative optimization algorithm significantly improves the main lobe energy concentration.

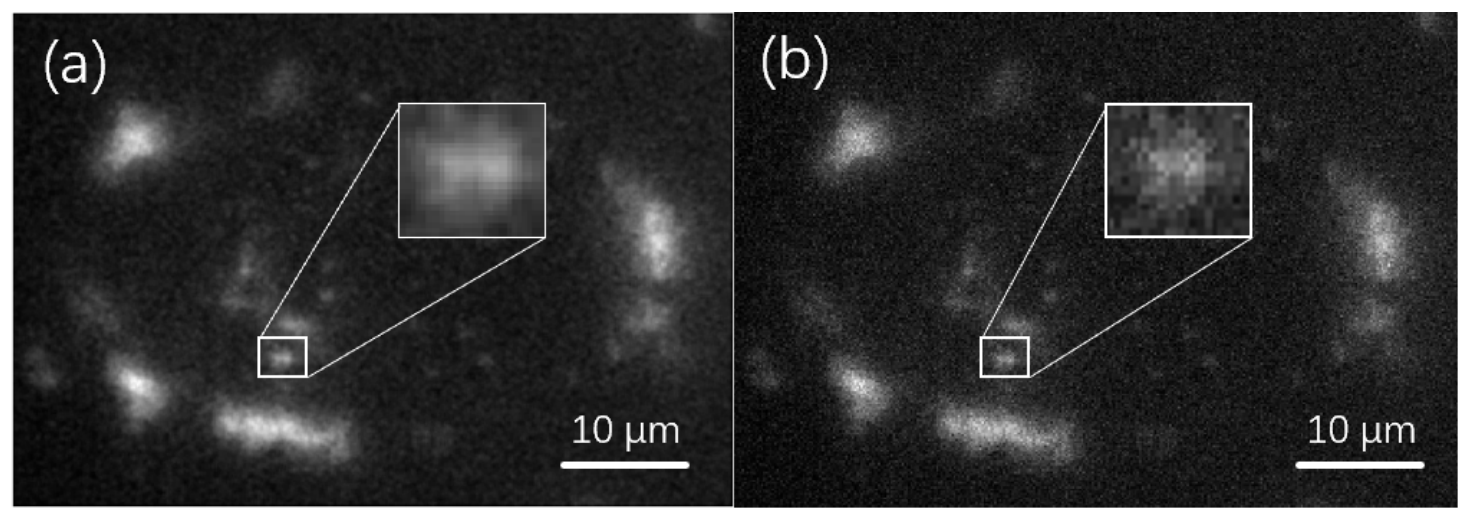

Figure 9. DH-PSF images of quantum dots in tumor cells: (a) before optimization; (b) after optimization.

\section{Conclusions}

In this work, an iterative optimization algorithm based on a Laguerre-Gaussian mode and Zernike polynomial is proposed, targeting the actual problems of the existing DH-PSF positioning systems and, specifically, the low concentration of the main lobe energy and the system's uncorrected aberration. The proposed scheme improves the main lobe energy concentration without affecting the double-helix characteristics of the phase modulation function. The simulation results showed that the average efficiency of the spot main lobe after the optimization process could be improved by $45.51 \%$, compared to its unoptimized counterpart. By building the experimental light-path and comparing the actual imaging effects of quantum dot labeled tumor cells, it was shown that the optimized image information entropy was reduced by $37.20 \%$ with respect to the unoptimized one. Thus, the image definition was significantly improved. The simulation experimental results strongly support that the proposed optimization process is of great importance for the improvement of the localization accuracy of fluorescent particles in cells and the in-depth study of tumor cell metabolic activities.

Author Contributions: Conceptualization, J.Y. and H.D.; methodology, J.Y. and H.D.; formal analysis, H.D.; investigation, H.D.; resources, J.Y., J.C., X.M., B.Q.L.; experimental operation, H.D., Z.C., L.Z.; data curation, H.D.; writing—original draft preparation, H.D.; writing—review and editing, J.Y. and H.D.; visualization, H.D.; funding acquisition, J.Y. All authors have read and agreed to the published version of the manuscript.

Funding: This work was financially supported by the National Natural Science Foundation of China (No. 51975465), the Key Research and Development Program of Shaanxi (No. 2020ZDLGY14-04), the China Postdoctoral Science Foundation (No. 2019M660251).

Institutional Review Board Statement: Not applicable.

Informed Consent Statement: Not applicable.

Data Availability Statement: The data presented in this study are available on request from the corresponding author.

Acknowledgments: The author would like to thank the fellow apprentices in the research group for their help.

Conflicts of Interest: The authors declare no conflict of interest. 


\section{References}

1. Carr, A.R.; Ponjavic, A.; Basu, S.; McColl, J.; Santos, A.M.; Davis, S.; Laue, E.D.; Klenerman, D.; Lee, S.F. Three-Dimensional Super-Resolution in Eukaryotic Cells Using the Double-Helix Point Spread Function. Biophys. J. 2017, 112, 1444-1454. [CrossRef]

2. Jeevanandam, J.; San Chan, Y.; Danquah, M.K. Nano-formulations of drugs: Recent developments, impact and challenges. Biochimie 2016, 128, 99-112. [CrossRef]

3. Champion, J.A.; Katare, Y.K.; Mitragotri, S. Particle shape: A new design parameter for micro- and nanoscale drug delivery carriers. J. Control Release 2007, 121, 3-9. [CrossRef]

4. Toy, R.; Peiris, P.M.; Ghaghada, K.B.; Karathanasis, E. Shaping cancer nanomedicine: The effect of particle shape on the in vivo journey of nanoparticles. Nanomedicine 2014, 9, 121-134. [CrossRef]

5. $\quad$ Dong, B.; Soetikno, B.T.; Chen, X.; Backman, V.; Sun, C.; Zhang, H.F. Parallel Three-Dimensional Tracking of Quantum Rods Using Polarization-Sensitive Spectroscopic Photon Localization Microscopy. ACS Photonics 2017, 4, 1747-1752. [CrossRef]

6. Opatovski, N.; Ezra, Y.S.; Weiss, L.E.; Ferdman, B.; Orange-Kedem, R.; Shechtman, Y. Multiplexed PSF Engineering for ThreeDimensional Multicolor Particle Tracking. Nano Lett. 2021, 21, 5888-5895. [CrossRef]

7. Yang, J.; Ling, Z.; Li, B.Q.; Li, R.; Mei, X. Nanoscale 3D temperature gradient measurement based on fluorescence spectral characteristics of the CdTe quantum dot probe. Opt. Express 2019, 27, 6770-6791. [CrossRef]

8. Hu, Y.; Li, S.; Kuang, C.; Xiu, P.; Ge, J.; Liu, X. An axial displacement measurement relying on the double-helix light beam. Opt. Laser Technol. 2014, 59, 1-6. [CrossRef]

9. Gustavsson, A.-K.; Petrov, P.N.; Lee, M.Y.; Shechtman, Y.; Moerner, W.E. 3D single-molecule super-resolution microscopy with a tilted light sheet. Nat. Commun. 2018, 9, 123. [CrossRef]

10. Shen, H.; Tauzin, L.J.; Baiyasi, R.; Wang, W.; Moringo, N.; Shuang, B.; Landes, C.F. Single Particle Tracking: From Theory to Biophysical Applications. Chem. Rev. 2017, 117, 7331-7376. [CrossRef]

11. Pavani, S.R.P.; Piestun, R. High-efficiency rotating point spread functions. Opt. Express 2008, 16, 3484-3489. [CrossRef]

12. Wang, Z.; Cai, Y.; Liang, Y.; Dan, D.; Yao, B.; Lei, M. Aberration correction method based on double-helix point spread function. J. Biomed. Opt. 2019, 24, 031005. [CrossRef]

13. Piestun, R.; Schechner, Y.Y.; Shamir, J. Propagation-invariant wave fields with finite energy. J. Opt. Soc. Am. A—Opt. Image Sci. Vis. 2000, 17, 294-303. [CrossRef]

14. Guo, C.S.; Liu, X.; He, J.L.; Wang, H.T. Optimal annulus structures of optical vortices. Opt. Express 2004, 12, 4625-4634. [CrossRef]

15. Kotlyar, V.V.; Kovalev, A.A.; Soifer, V.A.; Tuvey, C.S.; Davis, J.A. Sidelobe contrast reduction for optical vortex beams using a helical axicon. Opt. Lett. 2007, 32, 921-923. [CrossRef]

16. Chen, J.; Yuan, X.C.; Zhao, X.; Fang, Z.L.; Zhu, S.W. Generalized approach to modifying optical vortices with suppressed sidelobes using Bessel-like functions. Opt. Lett. 2009, 34, 3289-3291. [CrossRef]

17. Azucena, O.; Crest, J.; Kotadia, S.; Sullivan, W.; Tao, X.; Reinig, M.; Gavel, D.; Olivier, S.; Kubby, J. Adaptive optics wide-field microscopy using direct wavefront sensing. Opt. Lett. 2011, 36, 825-827. [CrossRef]

18. Azucena, O.; Crest, J.; Cao, J.; Sullivan, W.; Kner, P.; Gavel, D.; Dillon, D.; Olivier, S.; Kubby, J. Wavefront aberration measurements and corrections through thick tissue using fluorescent microsphere reference beacons. Opt. Express 2010, 18, 17521-17532. [CrossRef]

19. Schallek, J.; Geng, Y.; HoanVu, N.; Williams, D.R. Morphology and Topography of Retinal Pericytes in the Living Mouse Retina Using In Vivo Adaptive Optics Imaging and Ex Vivo Characterization. Investig. Ophthalmol. Vis. Sci. 2013, 54, 8237-8250. [CrossRef]

20. Jin, Y.; Chen, J.; Wu, C.; Chen, Z.; Zhang, X.; Shen, H.-1.; Gong, W.; Si, K. Wavefront reconstruction based on deep transfer learning for microscopy. Opt. Express 2020, 28, 20738-20747. [CrossRef]

21. Zhang, Y.; Zhou, T.; Fang, L.; Kong, L.; Xie, H.; Dai, Q. Conformal convolutional neural network (CCNN) for single-shot sensorless wavefront sensing. Opt. Express 2020, 28, 19218-19228. [CrossRef] [PubMed]

22. Qin, H. Aberration correction of a single aspheric lens with particle swarm algorithm. Opt. Commun. 2012, 285, 2996-3000. [CrossRef]

23. Bi, J.; Gao, Z.; Zhu, D.; Ma, J.; Yuan, Q.; Guo, Z.; Qu, Y.; Yin, C.; Xu, Y. An Optical Coherence Tomographic Aberration Correction Method Based on the Particle Swarm Optimization Algorithm. Acta Opt. Sin. 2020, 40, 1011002.

24. Ke, X.; Zhao, J. Analysis on characteristic of Laguerre-Gaussian beams with topological charges of arithmetic progression. Optik 2019, 183, 302-310. [CrossRef]

25. Kotlyar, V.V.; Khonina, S.N.; Kovalev, A.A.; Soifer, V.A.; Elfstrom, H.; Turunen, J. Diffraction of a plane, finite-radius wave by a spiral phase plate. Opt. Lett. 2006, 31, 1597-1599. [CrossRef]

26. Kotlyar, V.V.; Kovalev, A.A.; Skidanov, R.V.; Moiseev, O.Y.; Soifer, V.A. Diffraction of a finite-radius plane wave and a Gaussian beam by a helical axicon and a spiral phase plate. J. Opt. Soc. Am. Opt. Image Sci. Vis. 2007, 24, 1955-1964. [CrossRef]

27. Yang, J.; Ling, Z.; Li, R.; Mei, X. 3D localization at the nanometer scale and thermal sensing of living cells. J. Phys. Appl. Phys. 2019, 52, 365401. [CrossRef] 